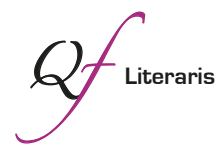

\title{
Novela inglesa y censura inquisitorial durante el reinado de Fernando VII: un expediente de 1815-1816
}

\author{
Begoña Lasa Álvarez \\ Universidade da Coruña \\ b.lasa@udc.es
}

Resumen: La traducción al castellano de la novela Memoirs of Miss Sydney Bidulph (1761) de la escritora Frances Sheridan fue censurada por el Tribunal del Santo Oficio, ya en los últimos años de vida de esta institución. El proceso se recoge en un expediente que se prolongó entre 1815 y 1816, aunque también contiene calificaciones de otro anterior desaparecido. Se trata de un expediente que se describe y se analiza en este trabajo por sus aportaciones en cuanto a los elementos que se consideraban censurables en un texto, así como por sus valiosas observaciones sobre el género novelesco, sobre la labor del traductor y sus condicionantes y, finalmente, sobre el papel que se atribuían los censores de tutores y guías de los lectores españoles.

Palabras clave: traducción; novela inglesa; censura; Inquisición; Frances Sheridan.

\begin{abstract}
The translation into Spanish of the novel Memoirs of Miss Sydney Bidulph (1761) by Frances Sheridan was censored by the Holy Office of the Inquisition in its final years of life. The whole process is registered in a record which lasted from 1815 to 1816 , although it also contains qualifications from a previously disappeared record. It is described and analysed in this article due to the data it contains regarding those elements considered reprehensible in a text, as well as for its valuable remarks on the narrative genre, on the translator's work and its determining factors and, finally, on the role assumed by the censors as tutors and guides of the Spanish readers.
\end{abstract}

Keywords: translation; English novel; censorship; Inquisition; Frances Sheridan.

》 Lasa Álvarez, Begoña. 2015. "Novela inglesa y censura inquisitorial durante el reinado de Fernando VII: un expediente de 1815-1816". Quaderns de Filologia: Estudis Literaris XX: 145-161. doi: 10.7203/qdfed.20.7534 



\section{Introducción}

"Sobre que se alce la suspensión de la venta de la obra en quatro tomos en octavo intitulada Memorias para la historia de la virtud". Con estas palabras comienza un extenso expediente inquisitorial que se inició el 19 de mayo de 1815 y finalizó el 23 de julio del año siguiente. En él se sometía a censura la traducción al castellano de la novela Memoirs of Miss Sydney Bidulph (1761) de la escritora de origen irlandés Frances Sheridan (1724-1766). Este texto se había publicado en cuatro tomos y sin nombre de autor bajo el título Memorias para la historia de la virtud, sacadas del diario de una señorita en 1792. Se indicaba que se había traducido del francés y, lo que es más reseñable en este caso, que se publicaba "Con licencia", es decir, que había pasado la censura previa gubernamental. Sin embargo, tras la restauración de Fernando VII y el restablecimiento de la censura, tanto gubernamental como inquisitorial, se produjo la suspensión de la venta de este texto y fue objeto de un largo proceso inquisitorial que aparece registrado en el expediente conservado en el Archivo Histórico Nacional con la referencia AHN, Inquisición, 4815/18. Por los documentos de que consta el mismo, la suspensión de la venta se había decretado con anterioridad, en 1807, pero a causa de la Guerra de la Independencia y de la supresión de la Inquisición por parte de Napoleón en diciembre de 1808 el expediente debió extraviarse o quedar en suspenso. Tras el restablecimiento de la Inquisición, el traductor, Jacobo de Villaurrutia, pide que se alce su prohibición.

El expediente ofrece interesantes aportaciones sobre los diversos aspectos en los que se centraban los censores de libros en el Tribunal de la Inquisición, destacando obviamente aquellos que se oponían a la religión católica, la única y verdadera fe en el estado confesional español. Como es sabido, este tribunal fue creado en 1478 por los Reyes Católicos precisamente para defender el catolicismo de todo tipo de herejía, en aquellos momentos principalmente el judaísmo y el islamismo, pero posteriormente también el luteranismo o protestantismo, como se puede apreciar en los argumentos de los censores de esta traducción. Además de este y otros elementos censurables sobre religión y sobre

\footnotetext{
* Este trabajo ha sido posible gracias al apoyo del Grupo de Investigación Cultura e Literatura Inglesa Moderna e Contemporánea (G000274) de la Universidade da Coruña.
} 
moral cristiana, el expediente que se analiza ofrece asimismo valiosas observaciones sobre el género novelesco, sobre la labor del traductor y sus diversos condicionantes por mor justamente del doble proceso de censura al que debía someter su trabajo y, lo que es más importante, sobre el papel de guardianes o tutores de los lectores que se arrogaban los censores, pues consideraban que los textos examinados o ciertos pasajes de los mismos no serían interpretados correctamente y por tanto podían inducir a error e incluso corromper las almas de los lectores, lo que a su vez justificaba y daba sentido a su trabajo.

Además de los estudios sobre censura e Inquisición, el análisis de este expediente se incluirá dentro del marco de los estudios de transferencia cultural, en los que procesos traductológicos como este, trilaterales o triangulares, tienen cabida y que se centran, más que en el mero producto de la traducción, en todo el proceso dentro de un contexto cultural determinado (Stockhorst, 2010: 19, 7), como es en este caso el de España en las primeras décadas del siglo XIX.

\section{Memoirs of Miss Sydney Bidulph y su recepción anterior en España}

Esta novela narra en forma epistolar la vida de Sydney Bidulph, una joven que tratando de comportarse virtuosamente rechazará a $\mathrm{Mr}$. Faulkland siguiendo el consejo materno, y por ello nunca encontrará la felicidad y sufrirá todo tipo de desgracias, al igual que su pretendiente, que terminará muriendo trágicamente. Al publicarse anónimamente ya en Inglaterra, cuando llegó a Francia y se tradujo al año siguiente tampoco se incluyó el nombre de la autora, lo que provocó que se atribuyera a su traductor, el abbé Prévost, que además había cambiado su título: Mémoirs pour servir à l'histoire de la vertu, extraits du journal d'une jeune dame ${ }^{1}$. A la vista del título es evidente que el traductor siguió la versión francesa para su traducción al castellano.

\footnotetext{
${ }^{1}$ En el título castellano jeune dame se traduce como señorita, un tratamiento que resultaba problemático, puesto que la protagonista contrae matrimonio en el transcurso de la novela. El inconveniente se solventa cambiando el título en los dos últimos volúmenes, pasando la señorita a ser señora. Sobre la recepción en España de esta novela, véase Lasa Álvarez (2009). También puede consultarse sobre la adaptación teatral de parte de esta novela a García Garrosa (2011).
} 
La práctica totalidad de textos ingleses que se traducían al castellano en estos momentos se realizaban desde el francés, y así sucedía también con los procedentes de otras lenguas como el alemán (Ruiz Casanova, 2000: 400; Lafarga, 2004: 210). Normalmente la educación de los individuos de las clases altas o medias-altas incluía el estudio del francés, por lo que muchos de ellos leían las novedades europeas en esta lengua; sin embargo, al democratizarse la lectura y alcanzar la educación a grupos sociales que anteriormente no habían podido acceder a ella, se hizo necesario traducir estos textos al castellano. De modo que lo habitual era que aquellos que se dedicaban a traducir lo hicieran desde el francés. Por otra parte, el hecho de que por esta vía llegaran a España novelas inglesas obedece al éxito general de la literatura inglesa y de la novela en particular, en Francia durante el siglo XVIII. Así, los lectores franceses esperaban con impaciencia las novedades procedentes de Inglaterra (Charles, 2003: 281). Del mismo modo, en España se puede hablar ya en los últimos años del setecientos de "una incipiente anglomanía, que se irá robusteciendo con los años, favorecida por razones de tipo político y militar en las primeras décadas del siglo XIX" (Lafarga, 2004: 210).

Como se ha mencionado más arriba, el texto pasó la censura gubernativa que actuaba sobre todo material impreso que se publicaba en España y que en la segunda mitad del siglo XVIII obedecía a una ley de 1751 y vigente hasta 1803. El procedimiento era el siguiente: el autor entregaba el ejemplar original al Consejo de Castilla y se nombraba un censor para realizar esta labor; una vez pasada la censura, si era positiva se le devolvía el libro a su autor para que este lo enviara a la imprenta, con la condición de que remitiera uno de los ejemplares al Consejo para su archivo, y lo mismo si había reimpresiones; la pena por incumplimiento de esta ley era la pérdida de bienes o el destierro (González Palencia, 1935: I, xiii-xiv; Aguilar Piñal, 2005: 182-183). Aunque la traducción del texto de Sheridan pasó con éxito este primer examen, muchas novelas de autores españoles y extranjeros fueron censuradas y prohibidas en España, como lo constatan los expedientes registrados por González Palencia (1935), Rumeu de Armas (1940) o Domergue (1980), entre los que se encuentran los emitidos a novelas inglesas (Pajares, 2010: 73-77). Por lo que se refiere a la censura inquisitorial, como señala Eterio Pajares, resultó una rémora atroz y en muchos casos actuó incluso de forma despótica con ciertos textos, como Joseph Andrews 
de Henry Fielding (2010: 77). Sin embargo, en este caso, puesto que ya había salvado el primer escollo y habían transcurrido ya más de 20 años desde su publicación, es comprensible que el traductor Jacobo de Villaurrutia se mostrara sorprendido por la censura de que fue objeto con la apertura del expediente del que a continuación se da cuenta.

\section{El expediente (AHN, Inquisición 4815/18)}

\subsection{Descripción general}

Se desconoce el desencadenante del proceso de censura de la traducción del texto de Sheridan, tanto en el primero de los expedientes, que por las fechas de los informes de los dos religiosos de Guatemala que aporta Villaurrutia ${ }^{2}$ en defensa de su traducción debió de comenzar en la primera mitad del año 1807, como en el caso del expediente que nos ocupa. Sin embargo, de entre las tres posibilidades que ofrecen Defourneaux (1973: 55) y Alcalá (2001: 14): los decomisos realizados en fronteras y navíos, las visitas a librerías o las denuncias de lectores escrupulosos, parece ser esta última la más plausible en el primero de los casos, al no tratarse esta obra de un texto conocido que pudiera ser identificado por el título. Por lo que se refiere al expediente iniciado en 1815 , podría tratarse del mismo traductor, quien decidiera resolver el asunto y no tener cuentas pendientes con este tribunal ${ }^{3}$. En cualquier caso, y a pesar de ciertas peculiaridades del expediente, el proceso que

\footnotetext{
${ }^{2}$ Jacobo de Villaurrutia, aunque había nacido en Santo Domingo en 1757, estudió en México y de allí se trasladó a España en el séquito del que luego sería cardenal de Toledo, Francisco Antonio Lorenzana (Méndez Reyes, 2001: 76). Como buen hombre de su tiempo, se interesó por el progreso, las reformas, la educación, y además de avanzar en su carrera profesional, tuvo tiempo para escribir de diversos temas, traducir y dedicarse al periodismo (ibid.: 77). Entre los puestos que ocupó, el mismo Villaurrutia menciona varios, pues cuando publicó la traducción era corregidor en Alcalá de Henares, posteriormente, cuando se produjo la primera censura de la traducción en 1807 era Alcalde del Crimen en Méjico, y en el momento en que se concluye el proceso era Oidor de la Real Audiencia de Barcelona.

${ }^{3}$ Más si cabe si tenemos en cuenta el Edicto de la Suprema y General Inquisición, publicado poco antes del inicio de este proceso, en abril de 1815, en el que se invitaba a descargar las conciencias de los pecadores concediéndoles un término de gracia hasta fin de ese año en el que si se autoinculpaban serían absueltos (García Cárcel \& Moreno, 2000: 101-102).
} 
se sigue y que a continuación se refiere coincide con los pasos descritos por Defourneaux (1973: 56-57).

El 19 de mayo de 1815 comienza el proceso cuando al Señor Inquisidor Fiscal se le envía una petición para que se alce la suspensión de la venta de la traducción en cuestión a instancias de su traductor, D. Jacobo Villaurrutia, cuya carta aparece como primer documento del expediente. En ella, como se verá, justifica algunos aspectos de su traducción y muestra su extrañeza ante la censura que recibió, con lo que se deduce que este expediente se produce como continuación de una censura anterior de la que no se aportan datos. Añade Villaurrutia que "desconfiando de sus cortas luces", pidió desde Méjico, donde se encontraba entonces, una respuesta a las censuras a dos religiosos de Guatemala, Antonio García Redondo y Fray José Antonio Goicoechea, unos documentos que también forman parte del expediente.

El 1 de junio el Inquisidor Fiscal Dr. Zorrilla de Velasco requiere al traductor para que presente un ejemplar de la obra que tradujo y las calificaciones anteriores, pues curiosamente no hay antecedentes ni ejemplar en ese tribunal. Al día siguiente, el Secretario de la Inquisición de Corte, D. Lorenzo Serrano, pide al traductor que le envíe un ejemplar de dicha obra, pues "por los trastornos pasados ha desaparecido el que existía en este Santo Oficio". Villaurrutia remite con el mismo portador el ejemplar solicitado que, según dice, "había mandado encuadernar y no envie [sic] con mi oficio anterior por un olvido natural"4.

Serrano envía los cuatro tomos de la obra junto con los dictámenes a favor y en contra ya realizados a un nuevo calificador, Miguel Antonio del Rincón, carmelita calzado en Madrid, que posteriormente se excusa por la tardanza, señalando que debía acabar antes otro dictamen de una obra que describe como de mayor importancia "sobre lo discutido y determinado en las Cortes relativo al Santo Tribunal". Esta observación de Rincón vendría a corroborar un aspecto señalado por los historiadores sobre el papel desempeñado por el Santo Oficio durante su restable-

\footnotetext{
${ }^{4}$ Los trastornos que Serrano menciona hacen referencia a los hechos que tuvieron lugar tras la publicación de los Decretos de Chamartín en noviembre de 1808, firmados por Napoleón, y por los que se eliminaba, entre otras instituciones del Antiguo Régimen, el Tribunal de la Inquisición (La Parra \& Casado, 2013: 80). Juan Antonio Llorente narra cómo el general de brigada francés Lauverdiere, comandante y gobernador de Madrid, se apoderó de las llaves de la sede central y permitió que durante dos meses se sacaran libros y papeles (1812: 9), perdiéndose por tanto mucha documentación.
} 
cimiento por Fernando VII, que era más político que religioso, pues su empeño se dirigió a censurar y descalificar las ideas liberales introducidas en España durante la Guerra de la Independencia y promovidas por las Cortes de Cádiz (Dufour, 1986: 118; La Parra \& Casado, 2013: 135136). Rincón envía finalmente el expediente de censura rubricado por él y por el doctor en Teología Manuel Andrés Luna, también carmelita calzado, en el que tras su valoración concluyen que puede levantarse la prohibición de la venta de la obra traducida, con la condición de que se borre una proposición que consideran herética. Junto a este documento se incluyen las otras calificaciones a las que se ha venido aludiendo en el expediente y que como se ha mencionado pertenecerían a otro anterior: dos censuras en contra sin fecha ni nombre de calificador y dos a favor, realizadas a requerimiento del traductor por dos religiosos y fechadas en octubre y diciembre de 1807 respectivamente.

Zorrilla de Velasco, al comprobar que los dictámenes son contrarios en lo sustancial, estipula que debe enviarse la obra y el resto de documentos del expediente a nuevos teólogos "que decidan la controversia”. Un nuevo calificador, Thomas Ozores de Puga, junto al Padre Juan Antonio Escalera, ambos religiosos de la Casa Profesa de los Padres Agonizantes de Madrid, remite un dictamen en el que se aprueba el levantamiento de la prohibición pero se solicita expurgación de un número considerable de pasajes.

El Consejo se reúne el 11 de mayo de 1816 y su disposición final se ajusta a la más leve de las censuras, pues resuelven que se alce la suspensión de venta y se le pide al traductor que únicamente explique en una nota el verdadero sentido de la siguiente proposición:

que las astucias de los malos deben combatirse con astucias, que la venganza es no solamente permitida, sino mandada á los buenos contra los pecadores (Sheridan, 1792: II, 273).

La consiguiente explicación de Villaurrutia en la nota dice:

que las astucias de los malvados no se han de dejar impunes para que estos queden triunfantes y aprovechados del fruto de su iniquidad, sino que se les debe contener, corregir y escarmentar con otras astucias: y esta es la especie de venganza de que se habla. 
Otro censor, Ildefonso Bueno, fraile benedictino, deberá indicar si le satisface o no y responde señalando que no basta la nota, sino que hay que borrar la proposición de las impresiones ya hechas y omitirla en las que se puedan realizar en un futuro. Decisión ratificada por el Consejo de 13 de julio de 1816. En oficio de 15 de julio se le transmite la providencia a Villaurrutia y el expediente acaba con una nota de este en la que comunica que da orden para que se realice lo resuelto por el tribunal.

\subsection{Sobre novela y traducción}

La traducción del texto de Sheridan no se vincula directamente al género novelesco, ya que aparece descrito en el título como memorias, un hecho bastante corriente a finales del siglo XVIII, pues se trataba de un género híbrido cuya adscripción los autores, y también traductores, trataban de evitar, así como falto de tradición, frente a los géneros de prestigio como la poesía y el teatro (Álvarez Barrientos, 1991: 27, 13). Por otra parte, no existía una terminología unificada para denominar a los diferentes géneros narrativos y su uso dependía en gran medida de la valoración y teoría que sobre la novela tuviera el autor (Checa Beltrán, 1992: 15). Así, aquellos escritores o traductores que quisieran publicar una novela en estos momentos, como bien manifestó Lucienne Domergue,

casi tenían que pedir perdón, o por lo menos pretender que el gusto sacado de su lectura solo servía para dar, más eficazmente, una lección moral al pueblo lector (1985: 486).

Por lo que el traductor de Memoirs of Miss Sidney Bidulph no deja de mencionar en su prólogo la utilidad del texto, aunque también añadirá otro paratexto, el prólogo de Prévost a su traducción al francés, en el que se ensalza la novela inglesa por ser el producto de una sociedad amante del orden y de las buenas costumbres (Lasa Álvarez, 2009: 684685). De todos los calificadores que forman parte de este expediente, solamente uno de ellos, el padre Miguel Antonio del Rincón, hace referencia al género literario del texto y pese a que se trata de unos comentarios realizados ya en la segunda década del XIX, la novela no merece buenas críticas como género y dice de ella despectivamente que de lo 
único que se ocupa es de la narración de eventos fantásticos e inverosímiles:

Nosotros suponemos que es una Novela, forjada como otras muchísimas que se han publicado en este tiempo novelero. ¿Quién sino la fantasía pudo servir de Correo para dirigir los Diarios de Madama Sydney a su amiga Cecilia? ¿Qué tiempo no se necesitaba para los acontecimientos tan raros que se refieren cuando la agudeza y oportunidad de un Calderón y de un Moreto acaso se vería en aprieto para su invención y colocación? Aunque se dice en la introducción que existían algunas personas interesadas en los sucesos que se refieren, nosotros no deseamos otro título que el de Novela, ni creeremos otra cosa.

Por lo que se refiere a la traducción, destacan las palabras del propio traductor en el oficio que remite al tribunal, en el que se refiere en ciertos pasajes a su labor como tal. Señala en primer lugar que realizó dicha traducción en los momentos de ocio que le permitía su trabajo como corregidor en Alcalá de Henares en aquel momento. Lo cierto es que muchas personas que habían recibido una educación y que atravesaban estrecheces económicas se dedicaban a traducir, fruto, como señala Ruiz Casanova,

de un modo de entender la traducción que es en parte medio de subsistencia o de complementar los ingresos y también labor mercenaria, mecánica o a la que se concede escasa importancia (2000: 401).

No sabemos con certeza el motivo por el que Villaurrutia decidió traducir esta obra, pero habitualmente quienes se dedicaban a traducir poseían otra profesión reconocida; y así, según un estudio llevado a cabo por García Hurtado sobre la profesión de los traductores en la segunda mitad del siglo XVIII y principios del XIX, tras religiosos, escritores, médicos y militares, aparecen los juristas con un porcentaje de 5,54\% del total (1999: 40-41).

Resulta comprensible que tras el arduo trabajo que suponía traducir un texto, y que en el caso de las novelas era de una extensión considerable, los traductores evitaran problemas con la censura y trataran de ver así publicado su trabajo (Pajares, 2010: 79). En el caso de Villaurrutia, al haber recibido ya la censura, reclama que se levante la sanción contra su traducción valiéndose de diferentes medios, como las calificaciones 
que en defensa de su texto escribieron los dos religiosos de Guatemala, y tratando de llevar a cabo cuanto antes las órdenes del tribunal cuando le solicitan que adjunte una nota explicativa al texto que finalmente consideran censurable. No obstante, Villaurrutia también previene la posible acción de la censura, y como muchos otros practicó la autocensura, pues, como indica en su oficio, supo que existía una segunda parte o continuación de esta novela, pero precisa que "conocí que eran de diversa mano y que no eran convenientes a nuestras costumbres: por lo cual hice animo a no traducirlos nunca"s. Uno de los calificadores, Ozores de Puga, refiriéndose a estas palabras del traductor, considera que en ellas se aprecian errores con relación a las reglas de la Vida Cristiana, pero los disculpa por ser una reacción lógica de Villaurrutia al ver "desairadas sus tareas" y por "el temor e incertidumbre que es capaz de inspirar un Expediente delicado", lo que demuestra que el calificador conocía el modo en que la existencia de la censura condicionaba a autores y traductores.

La censura de este último religioso es especialmente significativa en lo que se refiere al papel tutelar de los censores como guardianes de la fe y la moral de los lectores (Pajares, 2010: 63; Alcalá, 2001: 215), pues a su juicio no alcanzaban el nivel de instrucción necesario para entender ciertas expresiones, en particular "algunas proposiciones que en nuestro suelo podrán ser perjudiciales y ofender a los oídos piadosos". Más interesante para este trabajo es el hecho de que el calificador es consciente de que está juzgando un texto traducido y añade que

las citadas Memorias contienen algunas expresiones que a primera vista pueden presentarse bajo el aspecto de mal sonantes en nuestro País e Idioma especialmente a personas que no tienen motivo para darles otro significado y que no entienden ser frases expresivas del nativo en que fueron escritas.

Esta preocupación y celo por los receptores de los textos y sus consecuencias en la traducción, en especial la censura y autocensura, llevó a Eterio Pajares a calificar la traducción en este periodo como traducción tutelada (2010: 63).

\footnotetext{
${ }^{5}$ Efectivamente, como señala Villaurrutia, se publicó una segunda parte o secuela de este texto con el título de Conclusion of the Memoirs of Miss Sidney Bidulph en 1767. Sin embargo, fue escrita por la misma autora, Frances Sheridan.
} 


\subsection{Censuras y expurgaciones}

En primer lugar, debe tenerse en cuenta que las censuras dependían del talante individual de cada censor, y por tanto eran en cierto modo imprevisibles, lo que se traducía en una incertidumbre en los autores y traductores, que no podían prever el resultado (Alcalá, 2001: 15), y que se percibe en los escritos de Villaurrutia en el expediente. Lo que sí es cierto es que los delitos se fueron suavizando con el paso del tiempo (La Parra \& Casado, 2013: 141-142) y normalmente se reducían a proposiciones, es decir, "afirmaciones, dichos o expresiones interpretables en sentido no católico o heterodoxo" (ibíd.: 25), que son lo que vamos a encontrar en las diversas calificaciones de este expediente. Resultan elocuentes en este sentido algunas de las proposiciones reseñadas por el calificador Ozores de Puga para el tomo primero:

Pág. 37, aunque el caballero no trata de comparar a su Madre con la Virgen cuando la llama la mejor de las Mujeres, sino que se produce en estilo respetuoso en aquel País igual al que usan de la mejor de las Madres, son mal sonantes en el nuestro.

[...]

Pág. 222, proposición escandalosa que mirada en su primer y segundo significado contradice a los preceptos y máximas del Evangelio 6 .

Pág. 232, expresión mal sonante, y tomada en sentido rigoroso, impía, pues Dios solo es la Bondad misma, o esencialmente ${ }^{7}$.

En cualquier caso, y dependiendo de la gravedad de las mismas, se permitía la venta de la obra, se condenaba y prohibía in totum, o bien, quedaba prohibida hasta que se expurgaran sus proposiciones condenables (Defourneaux, 1973: 62; Alcalá, 2001: 16). La progresiva atenuación en las penas se observa en el expediente, pues si como consecuencia de lo afirmado por los primeros censores de 1807 se prohíbe la venta de la traducción, cuando se reinicia el expediente, los censores opinan que se puede levantar dicha prohibición y que la obra se venda con salvedades -el calificador Rincón pide que se borre una única proposición, mien-

\footnotetext{
${ }^{6}$ Se refiere con esta proposición a una frase del texto en la que un personaje declara que jamás podría perdonar a otro (Sheridan, 1792: I, 222).

${ }^{7}$ La proposición es consecuencia de unas palabras de la protagonista de la novela, que dice que "por fortuna mi madre es la misma Bondad" (Sheridan, 1792: I, 232).
} 
tras que Ozores de Puga ofrece una lista con un considerable número de proposiciones a expurgar, de las que ya se han ofrecido algunos ejemplos. Como ya se ha mencionado, la resolución del Consejo se inclinará por la más benévola de las dos y no ordena que se borre dicha proposición, sino que se añada una nota aclaratoria sobre su verdadero sentido. Aunque finalmente, tras la intervención de un último censor, se decidirá que la proposición deberá ser borrada en las impresiones ya realizadas y que se omita en las nuevas que se puedan publicar.

Dado el título de la novela en su traducción al castellano, el tema de la virtud se menciona en todas las censuras y de forma bastante detallada. En este sentido, en una de las dos censuras del primer expediente de 1807, la más rigurosa, se sostiene que "con mayor razón pudiera titularse Memorias para la historia del vicio". Posteriormente y en defensa de la novela, Fray J. Antonio Goicoechea señala sin embargo que al ser todos "criaturas frágiles y herederas del pecador Adán”, junto a las virtudes presentamos defectos y flaquezas, y menciona las historias de ejemplaridad cristiana de David y de Santa Teresa de Jesús, en las que se narran tanto sus actos virtuosos como las miserias en que incurrieron. Ya en 1815 el calificador Rincón señala que el título puede ser confuso, pues al mencionar la virtud, puede llevar al lector a creer que está ante una vida de santos o de algún asceta, y opina que un título más adecuado y menos problemático sería Memorias para la Historia de las ventajas que siguen a una buena crianza.

Otro aspecto sobre el que todos los censores ofrecen su parecer es la idoneidad o no de la presencia de personajes perversos en la novela. El traductor cree que es necesario ofrecer "el claro y obscuro de el cuadro" para que así resalten las prendas de los personajes con buenas cualidades. El padre Goicoechea abunda en este tipo de defensa aportando diversos ejemplos, como un sermón del teólogo y orador Bordalise, en el que

hace ver los frutos incomparables que sacan los buenos de los malos y como nos hacemos inexcusables delante de Dios de no habernos aprovechado del fruto que podemos sacar a vista de los pecadores y sus malos ejemplos,

o diversos episodios del viejo y nuevo testamento que, además de contener un gran número de casos virtuosos, se entremezclan con otros "horrendos de la perversidad de los hombres". El censor Rincón también 
está de acuerdo con que es necesario incluir malos ejemplos, pues "lo blanco junto a lo negro sobresale más".

En los diversos expedientes se alude a ciertos episodios de la novela que también pueden resultar perniciosos y que el primer calificador considera "ejemplos de libertinaje". Se refieren a relaciones adúlteras como la de Madama Gorin, que vive amancebada en París (Sheridan, 1792: IV, 164), o "el sospechoso procedimiento" del Coronel Smit y Madama Falklan, a quienes encuentra el marido de esta en la cama (Sheridan, 1792: IV, 293). El segundo calificador del primer periodo también se refiere a "asuntos e incentivos de amores entre jóvenes con detrimento de la sana moral", pues se hallan comprendidos en la regla séptima del Índice expurgatorio. Según esta regla, las obras que tratan sobre temas lascivos o de amores, aunque no se mezclen en ellas herejías o errores de este tipo, son dañosas para las costumbres de los buenos cristianos (Defourneaux, 1973: 50). Sin embargo, Rincón se muestra mucho más indulgente, aunque no sin ciertas pinceladas irónicas en sus comentarios, y sostiene que si se tienen en cuenta todos los elementos censurados por los calificadores anteriores "se hace preciso que el Sto Tribunal mande quemar los miles de libros y Folletos que hay en las Librerías y Casas".

Además de estos aspectos en cierto modo generales y que se podrían encontrar en censuras referidas a textos españoles, hay otros rasgos específicos que se relacionan con el lugar de origen del texto, como ocurre con las diferencias con respecto al celibato de los clérigos en la Iglesia católica y protestante. En un pasaje del texto de Sheridan un párroco aparece acompañado de su familia (Sheridan, 1792: I, 180). Los dos primeros censores se muestran incluso intolerantes, aduciendo que esta presencia "ultraja el celibato", y por otro lado señalan la necesidad de proteger a ciertos lectores, apelando a su rol de guardianes de la fe, especialmente a aquellos que no saben que en otras iglesias se permite el matrimonio de los sacerdotes, y que por tanto se podrían ofender. Goicoechea, en su defensa de la traducción, no considera este aspecto de tanta relevancia, pues no parece contemplar a los lectores tan inocentes o ignorantes: "nadie se escandaliza en el día de ver en los libros semejantes relaciones". Del mismo modo, Rincón en su expediente señala que "ningún mérito debe hacerse de que como Luterano fuese casado y con hijos". También se alude a otras diferencias culturales relativas a la religión, como cierto pasaje de la novela en el que a juicio de uno de 
los censores se satiriza contra los monasterios (Sheridan, 1792: I, 173; II, 411), o el mal ejemplo que ofrece uno de los personajes femeninos, Madama Gorin, que era católica y se convierte al protestantismo (Sheridan, 1792: II, 274).

Finalmente, resultan interesantes y curiosos ciertos aspectos un tanto banales del texto en los que los censores se detienen y discuten vehementemente, como es el caso de un fragmento del texto de Sheridan en el que la madre de Sydney le anima a que vaya al teatro y salga, incluso sin compañía (Sheridan, 1792: I, 6). También resulta prolija su explicación de la acepción del término adorar y de su correcta utilización, puesto que no están de acuerdo en si debería emplearse para describir el amor profano, como ocurre en la novela (Sheridan, 1792: I, 159), o si solo sirve para referirse a Dios. Por último, los censores también discrepan sobre si es lícito o no, de acuerdo con la religión católica, desear la propia muerte o la ajena (Sheridan, 1792: I, 86 y 275). Si los primeros censores se muestran totalmente escandalizados, en el caso de Goicoechea, como defensor, se muestra conforme en aquellas ocasiones en que de la muerte "nos resulta algún bien de superior orden respecto de la vida".

\section{Conclusión}

El expediente es una clara muestra de la actuación de la censura inquisitorial en sus últimos años de vida, pues se puede apreciar una importante diferencia en las calificaciones de los censores, con una progresiva suavidad en las penas, e incluso cierta falta de coherencia en su organización y en la aplicación de las reglas, aunque el fin perseguido sea el mismo, la tutela y protección de los lectores católicos españoles. También se observa la misma inconsistencia en la actuación del Consejo, que tras haber movilizado a varios censores, su decisión final es muy simple y no se aporta justificación alguna para la misma. Con respecto al género novelesco, no sale bien parado en las censuras, con lo que se aprecia un desfase entre la opinión de los calificadores y los gustos del público en general, un hecho que curiosamente uno de ellos admite con cierta ironía. Finalmente, y sobre la traducción, el expediente evidencia que los censores no tenían ningún reparo en traicionar el texto original en aras de la defensa de la fe verdadera, pues ese era su cometido principal, y es también reflejo de la incertidumbre y reservas con las que 
tenían que trabajar los traductores, pendientes como debían estar de la doble censura a la que se sometía su trabajo, y su impotencia ante las prohibiciones.

A pesar de todos estos impedimentos, en esta fase final de la Inquisición se puede hablar ya de una relajación del sistema (La Parra \& Casado, 2013: 10) y de la existencia de diversos modos de burlarla (Defourneaux, 1973: 73). En definitiva, dado el largo tiempo transcurrido desde su publicación, las consecuencias reales de este expediente para la novela en cuanto a su lectura y venta tuvieron que ser escasas. Así, a la vista de las diversas etapas por las que pasó el proceso, con tal cantidad de ir y venir de documentos, parece que se trató más de mantener el régimen burocrático establecido, que de conseguir que sus decisiones fuesen efectivas y se eliminase del texto todo aquello contrario a la fe católica. Además, Villaurrutia, un hombre que por sus diversos cargos estaba acostumbrado a lidiar con unos trámites administrativos que a cualquiera hubieran hecho cejar en el empeño, se mostró dispuesto a cumplir los requerimientos del tribunal y consiguió salir bastante airoso de las dificultades.

\section{Bibliografía}

Aguilar Piñal, Francisco. 2005. La España del Absolutismo Ilustrado. Madrid: Espasa-Calpe.

Álvarez Barrientos, Joaquín. 1991. La novela del siglo XVIII. Madrid: Júcar.

Alcalá, Ángel. 2001. Literatura y Ciencia ante la Inquisición Española. Madrid: Ediciones del Laberinto.

Charles, Shelly. 2003. Qu'est-ce qu'un roman anglais? D'Emma Courtney à La Chapelle d'Ayton. Eighteenth-Century Fiction 15(2): 281-301.

Checa Beltrán, José. 1992. Novela y teoría española dieciochista. Ínsula 546: 15-17.

Defourneaux, Marcelin. 1973. Inquisición y censura de libros en la España del siglo XVIII. Madrid: Taurus.

Domergue, Lucienne. 1985. Ilustración y novela en la España de Carlos IV. En Iglesias, M. ${ }^{a}$ Carmen; Moya, Carlos y Rodríguez Zúñiga, Luis (ed.) Homenaje a José Antonio Maravall. Vol I. Madrid: Centro de Investigaciones Sociológicas, 483-498.

Dufour, Gerard. 1986. La Inquisición española. Aproximación a la España intolerante. Barcelona: Montesinos.

García Cárcel, Ricardo y Moreno, Doris. 2000. Inquisición. Historia crítica. Madrid: Temas de Hoy. 
García Garrosa, María Jesús. 2011. Gaspar Zavala y Zamora y la sociedad inglesa. "El amante honrado": adaptación dramática de la novela $\mathrm{Me}$ moirs of Miss Sidney Bidulph. Dieciocho 34(1): 7-28.

García Hurtado, Manuel-Reyes. 1999. La traducción en España, 1750-1808: Cuantificación y lenguas en contacto. En Lafarga, Francisco (ed.) $L a$ traducción en España (1750-1830). Lengua, literatura, cultura. Lleida: Universitat de Lleida, 35-43.

González Palencia, Ángel. 1935. Estudio histórico de la censura gubernativa en España 1800-1833. 2 vols. Madrid: Tipografía de Archivos.

La Parra, Emilio y Casado, María Ángeles. 2013. La Inquisición en España. Agonía y abolición. Madrid: Los libros de la catarata.

Lafarga, Francisco. 2004. El siglo XVIII, de la Ilustración al Romanticismo. En Lafarga, Francisco y Pegenaute, Luis (ed.) Historia de la traducción en España. Salamanca: Ed. Ambos Mundos, 209-319.

Lasa Álvarez, Begoña. 2009. "La novela inglesa del siglo XVIII en España: el caso de Memorias para la historia de la virtud de 1792". En De Lorenzo, Elena (ed.) La época de Carlos IV (1788-1808). Oviedo: Ed. Trea, 677-686.

Llorente, Juan Antonio. 1812. Memoria histórica sobre qual ha sido la opinión nacional de España acerca del Tribunal de la Inquisición. Madrid: Imprenta de Sancha.

Méndez Reyes, Salvador. 2001. Las élites coloniales de la Nueva España y Chile ante la Independencia (tesis doctoral). México: UNAM. [Tesis digitales UNAM]. http://132.248.9.195/pd2001/294318/Index.html

Pajares, Eterio. 2010. La traducción de la novela inglesa del siglo XVIII. Vitoria: Portal Education.

Ruiz Casanova, José Francisco. 2000. Aproximación a una historia de la traducción en España. Madrid: Cátedra.

Rumeu de Armas, Antonio. 1940. Historia de la censura literaria gubernativa en España. Madrid: M. Aguilar.

Sheridan, Frances. 1792. Memorias para la historia de la virtud, sacadas del diario de una señorita. Tomos I y II. Alcalá: Imprenta de la Real Universidad.

Sheridan, Frances. 1792. Memorias para la historia de la virtud, sacadas del diario de una señora inglesa. Tomos III y IV. Alcalá: Imprenta de la Real Universidad.

Stockhorst, Stefanie. 2010. Introduction. Cultural transfer through translation: a current perspective in Enlightenment studies. En Stockhorst, Stefanie (ed.) Cultural Transfer through Translation. The Circulation of Enlightened Thought in Europe by Means of Translation. Amsterdam \& New York: Rodopi, 7-26. 
